\title{
Admission Characteristics and Outcomes of ED Patients With Rhabdomyolysis
}

\author{
Yalcin Golcuk ${ }^{1 *}$, Burcu Golcuk ${ }^{2}$, Murat Ozsarac ${ }^{1}$, Mehmet Irik ${ }^{1}$, Ayhan Korkmaz ${ }^{3}$, \\ Adnan Bilge ${ }^{1}$
}

\section{Abstract}

Objective: Rhabdomyolysis is a potentially life-threatening syndrome characterized by muscle necrosis and the release of potentially toxic intracellular muscle constituents into the circulation. Acute kidney injury (AKI) is the most important complication of rhabdomyolysis and is associated with increased risk of all-cause mortality. The main objectives of this study were (1) to characterize the most common etiologies of rhabdomyolysis and (2) to determine the complications and outcomes (rhabdomyolysis-induced AKI, need for renal replacement therapy [RRT] and, 28-day mortality) in our sample.

Material and Methods: This retrospective, cross sectional and single-center study was conducted in the ED of university hospital between January 1, 2013, and December 31, 2013. We analyzed the clinical spectrum and evaluated the complications and outcomes for each patient.

Results: Forty-three eligible cases were enrolled in the study. The mean age was $52.0 \pm 21.9$ years (range 16 to 92 ), and $81.4 \%$ were men. The two most common causes of rhabdomyolysis in this sample were trauma and infections $(n=16 ; 37.2 \%$ and $n=12 ; 27.9 \%$, respectively). AKI occurred in 23 patients $(53.4 \%), 13$ of whom $(30.2 \%)$ required RRT. All-cause 28-day mortality rate was $44.2 \%(n=19)$. The nonsurvival group had significantly increased peak creatinine level, increased phosphate level, and prolonged aPTT $(\mathrm{P}<.001, \mathrm{P}=.003$, and $\mathrm{P}=.001$, respectively).

Conclusion: A substantial proportion of patients with rhabdomyolysis developed the complications of AKI and required RRT. Early recognition and aggressive fluid replacement should be considered for ED patients with rhabdomyolysis.

Key words: Rhabdomyolysis, acute kidney injury, renal replacement therapy

\section{Introduction}

Rhabdomyolysis is a potentially lifethreatening syndrome characterized by muscle necrosis and the release of potentially toxic intracellular muscle constituents, including creatine phosphokinase $(\mathrm{CK})$, myoglobin $(\mathrm{Mb})$, electrolytes, lactate dehydrogenase, aldolase, alanine aminotransferase, and aspartate aminotransferase into the blood circulation (1-7).

There are numerous potential causes that can lead to rhabdomyolysis (eg, trauma and muscle compression, infections, myotoxins and drugs, marked exertion, prolonged immobilization, metabolic disorders, endocrine disorders, genetic disorders, hypothermia or malignant hyperthermia, seizure, acute extremity compartment syndrome, high-voltage electrical injury, or electrolyte abnormalities) (1-11). Although the causes are multiple, the final common pathway for injury is an increase in intracellular free

ionized cytoplasmic and mitochondrial calcium. This may be caused by depletion of ATP, the cellular source of energy, and/or by direct injury and rupture of the plasma membrane and the release of aforementioned muscle constituents into the blood circulation $(5,12)$.

Acute kidney injury (AKI) is the most important and serious complication of rhabdomyolysis independently of its etiology $(12,13)$. Although it is widely accepted that $\mathrm{AKI}$ is caused by $\mathrm{Mb}$ deposition in the kidney, the mechanism by which it occurs is not clearly understood. The current consensus is that AKI is due to the combined effects of hypovolemia, aciduria, and direct cytotoxicity due to accumulation of renal tubular $\mathrm{Mb}(8,12-15)$. It has been reported that $10-40 \%$ of patients with rhabdomyolysis develop AKI, and that $5-15 \%$ of cases of AKI are attributable to rhabdomyolysis $(9,13)$ Especially, the risk of

Received:31-08-2015, Accepted 19-09-2015, Available Online 28-02-2016

1Celal Bayar University, Medical Faculty, Dept. of Emergency Medicine, Manisa, TURKEY

2Merkez Efendi State Hospital, Dept. of Clinical Biochemistry, Manisa, TURKEY

3Merkez Efendi State Hospital, Dept. of Emergency Medicine, Manisa, TURKEY

*Corresponding Author: Yalcin Golcuk E-mail: dryalcingolcuk@gmail.com 
rhabdomyolysis-induced AKI is higher in patients with CK levels at admission more than $5000 \mathrm{IU} / \mathrm{L}$ (16).

Although many case reports appear in the literature on rhabdomyolysis from Turkey, to the best of our knowledge, this is the first study to analysed the clinical spectrum, prevalence of various etiologies, and explored the frequency of AKI in adult patients with rhabdomyolysis that presented to the emergency department (ED) $(17,18)$. The two main goals of this study were as follows: 1) to characterize the most common etiologies of rhabdomyolysis in our ED patients, 2) to determine the rhabdomyolysis-induced AKI, treatment modalities, and mortality in this sample. We believe that the present study will be useful and salutary for the progress of literature

\section{Material and Methods}

\section{Study design and setting}

This retrospective, single-center, crosssectional study was conducted at the ED of university hospital (Manisa, Turkey) between January 1, 2013, and December 31, 2013. The annual number of adult ED patient visits is approximately 40,000 per year. Upon approval from the local ethics committees (Reference number 20478486-53), this study was conducted according to the Principles of the Declaration of Helsinki of 1975, as revised in 1983. The need to obtain written consent informs was waived because of the retrospective nature of the study.

\section{Data collection and patient selection}

This study was a medical record review of patients 16 years or older who presented to the ED with a diagnosis of rhabdomyolysis based on their medical histories and elevated serum CK levels within 72 hours after admission to the ED. We identified potentially eligible patient visits by searching the hospital patient records database. Furthermore, the hospital information system database was also searched to identify all of the serum CK levels above $5000 \mathrm{IU} / \mathrm{L}$. We excluded patients who had 1) an age younger than 16 years 2) an elevated CK levels associated with an acute coronary syndrome (STelevation or non-ST-elevation myocardial infarction), 3) rhabdomyolysis that developed after admission to the hospital because of a coexisting condition or iatrogenic complication, 4) end-stage renal disease requiring haemodialysis or continuous ambulant peritoneal dialysis, 5) a cerebral vascular infarction or bleeding, and 6) a documented history of muscular dystrophy or other metabolic muscle disorder.

From all patients, the following variables were collected: age, gender, past medical history (epilepsy, hypertension, diabetes, history of renal insufficiency), medication use, recent physical exertion, recent trauma (crush injury, motor vehicle collision, fall, assault, burn, electrical injury), seizure, immobilization, dehydration, viral/bacterial illness, metabolic derangements (diabetic/ alcoholic ketoacidosis), hyper- or hypothermia, illicit drug use, vital parameters, therapeutic interventions, and length of stay (LOS) at the ED or hospital. In addition, we also obtained patients' clinical laboratory test results (initial and peak serum CK levels, white blood cell count, haemoglobin, platelet count, electrolytes, blood urea nitrogen, initial and peak serum creatinine, lactic dehydrogenase, alanine and aspartate aminotransferase, coagulation tests, and arterial blood gas values). Complications and outcomes (AKI, need for dialysis, and 28-day mortality) were recorded for each patient.

The concentrations of serum CK (reported as IU/L) were analysed using the Beckman Coulter chemiluminescent immunoassay on the Beckman Coulter Unicell DXC 800 immunoassay analyser. All serum CK values above the upper limit (1200 IU/L) were routinely assayed by dilution (10-fold), making quantitative assessment possible. The baseline serum $\mathrm{CK}, \mathrm{CK}-\mathrm{MB}$, and creatinine value was obtained on admission in the ED; the peak serum CK, CK-MB, and creatinine value was considered as the highest level during the ED or hospital stay. In our ED laboratory, serum and urinary $\mathrm{Mb}$ are not routinely assessed.

\section{Definitions}

Rhabdomyolysis classically defined as a CK levels greater than five times the upper limit of normal (approximately 850-1000 IU/L) (9-19). In our study, patients with rhabdomyolysis were defined as new proposed diagnostic criteria having absolute CK levels $>15.000 \mathrm{IU} / \mathrm{L}$ or CK levels $>5000 \mathrm{IU} / \mathrm{L}$ and any of the following: 1) crush injury, 2) AKI or overt failure, 3) myoglobinuria, 4) acidosis, disseminated intravascular coagulation (DIC), hypocalcemia, or hyperkalemia, 5) massive muscle injury, 6) prolonged extrication or initial evaluation delayed longer than 4 hours (20). Patients with acute kidney injury were defined as the abrupt ( $\leqslant 48$ hours) reduction of kidney function: increased serum creatinine levels (absolute, $\geqslant 0.3 \mathrm{mg} / \mathrm{dl}$; percentage, $\geqslant 50 \%$; or 1.5 fold from baseline), or oliguria $(<0.5 \mathrm{ml} / \mathrm{kg} / \mathrm{h}$ for more than 6 hours) by using the Acute Kidney Injury Network (AKIN) criteria (21). Renal replacement therapy (RRT) was defined as the use of peritoneal dialysis, haemodialysis or continuous renal replacement therapy. Indication for emergency RRT was determined by the consultant nephrologists.

\section{Statistical analysis}

All the patients were divided into two groups (survivors and non-survivors) based on their 28-day mortality. Continuous variables were expressed as mean $\pm \mathrm{SD}$ or median (interquartile range [IQR]) 
according to normal or non-normal distributions. Categorical variables were presented as absolute values and percentages. The normality of data distribution was checked with Shapiro-Wilks test. Group differences in categorical variables were compared using the Pearson $\chi 2$ test. Differences in continuous variables were evaluated by the MannWhitney $\mathrm{U}$ test. For all tests, $\mathrm{P}<.05$ was considered statistically significant. Statistical analyses were performed using SPSS software (version 20.0; SPSS Inc, Chicago, IL, USA).

\section{Results}

We identified 64 consecutive patient visits with an ED diagnosis of rhabdomyolysis over the 1year study period. Twenty-one patients were excluded (9 had rhabdomyolysis develop only after admission as a result of another disorder; 6 cases of rhabdomyolysis occurred in patients younger than 16 years; 3 were transferred another hospital; 2 were missing data; and 1 patient on chronic dialysis). Thus, 43 patients met the inclusion criteria and comprised the study population.

In those patients with an ED diagnosis of rhabdomyolysis, the mean age was $52.0 \pm 21.9$ years (range 16 to 92 ), and $81.4 \%$ were men. Table 1 summarizes the causes of rhabdomyolysis of patients in this study. The two most common causes of rhabdomyolysis in this sample were trauma and infections $(n=16 ; 37.2 \%$ and $n=12 ; 27.9 \%$, respectively). Baseline demographic, clinical, and laboratory characteristics of the patients were described and divided according to the survival status at 28 days in Table 2 . All the patients included in the study were hospitalized, total of $23(53.4 \%)$ patients developed AKI, and 13 (30.2\%) cases with AKI were treated with RRT. Rest of the patients treated with conservative treatment. All-cause 28-day mortality rate was $44.2 \% \quad(n=19)$. Mortality was significant higher among patients who developed AKI then in patients without AKI, $\mathrm{P}<.001$. Only 1 patient with AKI who treated with RRT discharged from the hospital with a full recovered kidney function. In addition, there was significant difference in terms of need for RRT between survivors and non-survivors groups, $\mathrm{P}<.001$. And also, the most common cause requiring the use of RRT was infections $(n=5$; $11.6 \%$ ). The median hospital LOS was 5 days (IQR, 1-80) and there was no significant difference between the means of the two groups.

With regard to the admission and peak serum CK levels, there were no significant difference between the survivors and non-survivors groups (7890 \pm 7353 vs. $9721 \pm 19267, \mathrm{P}=.282$ and $12778 \pm 8246$ vs. $20515 \pm 28144, \mathrm{P}=.751)$. Patients who did not survived had significantly increased peak creatinine level $(4.5 \pm 2.0$ vs. $1.7 \pm 1.4, \mathrm{P}<.001)$, increased phosphate level $(5.4 \pm 3.0$ vs. $3.1 \pm 0.9, \mathrm{P}=.003)$, and prolonged aPTT $(35.2 \pm 13.8$ vs. $24.6 \pm 8.2, \mathrm{P}=.001)$.

Discussion

As far as we know, this is the first clinical investigation of patients with rhabdomyolysis that presented to the ED in Turkey. In this study, we analyzed the prevalence of various etiologies, explored the frequency of AKI, and compared the demographic, clinical and, laboratory characteristics of the survivor and non-survivors adult patients with rhabdomyolysis.

The underlying causes of rhabdomyolysis are highly variable from hospital to hospital and, from country to country $(22,23)$. In our study, trauma accounted for more than one-third $(37.2 \%)$ of the causes of rhabdomyolysis. In the United States, data from the National Hospital Discharge Survey in 1995 , the main underlying cause of rhabdomyolysis is alcohol intoxication, followed by illicit drug use (24). However, in the recently larger adult study from United States conducted by McMahon et al identified 2371 patients and they found the main causative factor of rhabdomyolysis was trauma (25). In another important retrospective study from Spain performed by Rodriguez et al, the researchers examined 126 patients with severe rhabdomyolysis (defined as serum CK level > $5000 \mathrm{IU} / \mathrm{L}$ ), the most frequent cause was prolonged immobilization due to consumption of illicit drugs abuse (16). Interestingly, rhabdomyolysis due to illicit drugs abuse were not observed in our study of ED patients. Furthermore, in contrast to adult studies, the leading cause of rhabdomyolysis in pediatric patients is viral myositis $(26,27)$. On the other hand, traumatic injuries are the leading cause of youth adult's morbidity and mortality in the United States and Turkey (28-32). Therefore, all emergency physicians should be aware of the association between trauma and the risk of rhabdomyolysis.

Electrolyte abnormalities are the most feared complications and are common in patients with rhabdomyolysis. Hyperphosphatemia, one of them, results from the release of inorganic phosphate from damaged muscle cells. The subsequent development of AKI and acidosis can also lead to further increases in serum phosphate. Hyperphosphatemia has been shown to be a powerful risk factor for either predicting renal failure or death in rhabdomyolysis. In our study, with regard to the admission phosphate levels, there was significant difference between the survivors and non-survivors groups. Recently, a large study conducted by McMahon et al showed that hyperphosphatemia was associated with increased risk of developing AKI and mortality even when it was mild degree (25). It remains unclear whether the relationship between hyperphosphatemia and adverse outcomes is causative or only associative. 
Table 1. Causes of rhabdomyolysis

\begin{tabular}{lc}
\hline Causes & Study population $(\mathbf{n}=\mathbf{4 3})$ \\
\hline Trauma & 16 \\
Infections (sepsis or septic shock) & 12 \\
Vascular occlusion (embolism or thrombosis) & 5 \\
Toxins & 2 \\
Seizures & 2 \\
Malignant hyperthermia & 1 \\
Ethanol withdrawal & 1 \\
Metabolic disorders & 1 \\
Recent abdominal surgery & 1 \\
Inflammatory myopathy & 1 \\
Idiopathic & 1 \\
\hline
\end{tabular}

Table 2. Demographic, clinical and laboratory characteristics of patients with rhabdomyolysis

\begin{tabular}{|c|c|c|c|}
\hline Variables & Survivors $(n=24)$ & Nonsurvivors $(n=19)$ & $P$ value \\
\hline \multicolumn{4}{|l|}{ Demographic and clinical data } \\
\hline Age (y) & $46.3 \pm 23.0$ & $59.1 \pm 18.6$ & .056 \\
\hline Sex (Female/Male) & $4 / 20$ & $4 / 15$ & .507 \\
\hline AKI (\%) & $5(11.6)$ & $18(41.8)$ & $<.001$ \\
\hline RRT (\%) & $1(2.3)$ & $12(27.9)$ & $<.001$ \\
\hline Hospital LOS (days) & $10.9 \pm 15.9$ & $7.3 \pm 7.9$ & .296 \\
\hline \multicolumn{4}{|l|}{ Hematology profile } \\
\hline White blood cell count $\left(\times 10^{3} / \mu \mathrm{L}\right)$ & $13.8 \pm 6.8$ & $15.2 \pm 6.8$ & .501 \\
\hline Hemoglobin $(\mathrm{g} / \mathrm{dL})$ & $12.7 \pm 1.9$ & $11.6 \pm 2.2$ & .080 \\
\hline Hematocrit (\%) & $37.2 \pm 6.1$ & $34.7 \pm 5.2$ & .151 \\
\hline Platelet count $\left(\times 10^{3} / \mu \mathrm{L}\right)$ & $200.6 \pm 82.2$ & $169.1 \pm 91.2$ & .242 \\
\hline \multicolumn{4}{|l|}{ Coagulation profile } \\
\hline INR level & $1.0 \pm 0.1$ & $1.8 \pm 1.4$ & .011 \\
\hline PT (s) & $12.5 \pm 1.8$ & $20.9 \pm 16.9$ & .015 \\
\hline aPTT (s) & $24.6 \pm 8.2$ & $35.2 \pm 13.8$ & .001 \\
\hline \multicolumn{4}{|l|}{ Serum chemistry } \\
\hline Glucose (mg/dL) & $152.5 \pm 74.1$ & $159.6 \pm 94.5$ & .590 \\
\hline Blood urea nitrogene $(\mathrm{mg} / \mathrm{dL})$ & $30.5 \pm 34.6$ & $48.5 \pm 39.6$ & .117 \\
\hline Uric acit (mg/dL) & $6.0 \pm 1.9$ & $9.0 \pm 6.1$ & .065 \\
\hline Admission creatinine $(\mathrm{mg} / \mathrm{dL})$ & $1.4 \pm 0.7$ & $1.7 \pm 1.0$ & .031 \\
\hline Peak creatinine $(\mathrm{mg} / \mathrm{dL})$ & $1.7 \pm 1.4$ & $4.5 \pm 2.0$ & $<.001$ \\
\hline Sodium $(\mathrm{mEq} / \mathrm{L})$ & $136.6 \pm 6.3$ & $136.9 \pm 5.1$ & .941 \\
\hline Potassium $(\mathrm{mEq} / \mathrm{L})$ & $4.2 \pm 0.6$ & $4.7 \pm 1.1$ & .276 \\
\hline Chloride $(\mathrm{mEq} / \mathrm{L})$ & $104.1 \pm 6.9$ & $102.5 \pm 5.7$ & .225 \\
\hline Calcium (mg/dL) & $8.5 \pm 0.8$ & $8.1 \pm 1.0$ & .107 \\
\hline Phosphate (mg/dL) & $3.1 \pm 0.9$ & $5.4 \pm 3.0$ & .003 \\
\hline Magnesium (mg/dL) & $1.9 \pm 0.2$ & $2.3 \pm 0.6$ & .006 \\
\hline Admission CK (IU/L) & $7890 \pm 7353$ & $9721 \pm 19267$ & .282 \\
\hline Peak CK (IU/L) & $12778 \pm 8246$ & $20515 \pm 28144$ & .751 \\
\hline Admission CK-MB (IU/L) & $233 \pm 237$ & $316 \pm 562$ & .549 \\
\hline Peak CK-MB (IU/L) & $375 \pm 372$ & $507 \pm 624$ & .980 \\
\hline Total protein $(\mathrm{g} / \mathrm{dL})$ & $6.1 \pm 1.1$ & $5.9 \pm 0.9$ & .677 \\
\hline Albumin $(\mathrm{g} / \mathrm{dL})$ & $3.4 \pm 0.7$ & $3.1 \pm 0.5$ & .086 \\
\hline Aspartate transaminase (IU/L) & $203.5 \pm 174.7$ & $424.2 \pm 774.6$ & .608 \\
\hline Alanine transaminase (IU/L) & $116.7 \pm 124.3$ & $251.8 \pm 445.0$ & .633 \\
\hline \multicolumn{4}{|l|}{ Blood gas analysis (arterial) } \\
\hline $\mathrm{pH}$ (pH units) & $7.36 \pm 0.06$ & $7.25 \pm 0.21$ & .084 \\
\hline $\mathrm{HCO}_{3}^{-}(\mathrm{mmol} / \mathrm{L})$ & $22.3 \pm 3.9$ & $18.4 \pm 5.4$ & .009 \\
\hline Lactate $(\mathrm{mmol} / \mathrm{L})$ & $2.7 \pm 2.9$ & $5.2 \pm 4.5$ & .019 \\
\hline $\mathrm{BE}^{\mathrm{ecf}}(\mathrm{mmol} / \mathrm{L})$ & $-3.3 \pm 3.3$ & $-7.6 \pm 8.2$ & .082 \\
\hline Anion Gap $(\mathrm{mmol} / \mathrm{L})$ & $2.9 \pm 4.9$ & $3.1 \pm 5.6$ & .905 \\
\hline
\end{tabular}

Data are expressed as mean \pm SD or count (percentage of the 43 subjects) for categorical variables unless otherwise indicated.

Abbreviations: AKI, Acute Kidney Injury; RRT, Renal Replacement Therapy; LOS, Length of Stay; INR, International Normalized Ratio; PT, Prothrombin Time; aPTT, Activated Partial Thromboplastin Time; s, Seconds; CK, Creatine Kinase; CK-MB, Creatine Kinase-MB; pH, power of Hydrogen; BEecf, Base Excess of extracellular fluid 
Coagulation studies (PT, aPTT, fibrin split products and fibrinogen) should be obtained in all suspected cases of rhabdomyolysis. Because, severe rhabdomyolysis may be associated with the development of disseminated intravascular coagulation (DIC) due to activation of the cytokine network and the release of thromboplastin and other prothrombotic substances from the damaged muscle. DIC is more frequently observed in patients with posttraumatic rhabdomyolysis with manifesting the systemic inflammatory response syndrome. Rodriguez et al found that decreased PT can confer a 4.4-fold increased risk for development of the AKI in study population (16). In our study, prolonged aPTT was found associated with increased risk of mortality.

AKI is a serious and often life-threatening complication of rhabdomyolysis and requires immediate diagnosis and emergency treatment $(9,33,34)$. AKI is believed to be due to decreased extracellular volume, which results in renal vasoconstriction. It is also believed to be due to ferrihemate, which is formed from $\mathrm{Mb}$ at a $\mathrm{pH}$ level of 5.6 or less. Ferrihemate produces free hydroxy radicals and causes direct nephrotoxicity, often through lipid peroxidation $(2,5,12,13)$. Previous studies on rhabdomyolysis have reported rates of AKI with or without the need for RRT ranging from 14$59 \%$ in adults $(9,35)$. In all these studies various population settings and different definitions of AKI have been used. Consequently, the exact incidence of AKI is difficult to determine. Delaney et al recently conducted a retrospective study showing that based on the RIFLE criteria, $59 \%$ of the patients with rhabdomyolysis had AKI, most of which were prerenal.35 However, a study by Chen et al found that only $14.4 \%$ patients with rhabdomyolysis developed AKI (9). In a recent study of 521 pediatric patients with posttraumatic rhabdomyolysis, AKI occurred in $70(13.4 \%)$ patients (36). In our study based on the AKIN criteria, total of $23(53.4 \%)$ patients developed AKI. This suggests that various factors and conditions may play a role in the development of the AKI in patients with rhabdomyolysis.

Currently, there is no way to accurately predict or stratify mortality risk or risk of AKI among patients with rhabdomyolysis. Several studies have attempted to predict the likelihood of AKI by means of biochemical and clinical markers. In a recent study, McMahon et al developed a new risk score for predicting death or AKI in rhabdomyolysis. The independent predictors of death or AKI were age (5070 years, 1.5 points; 71-80 years, 2.5 points; $>80$ years, 3 points), female sex (1 point), cause of rhabdomyolysis (origin not seizures, syncope, exercise, statins, or myositis, 3 points), and values of initial creatinine (1.4-2.2 $\mathrm{mg} / \mathrm{dL}, 1.5$ points; $>2.2$ $\mathrm{mg} / \mathrm{dL}, 3$ points), $\mathrm{CK}$ (>40.000 U/L, 2 points), phosphate (4.0-5.4 mg/dL, 1.5 points; $>5.4 \mathrm{mg} / \mathrm{dL}, 3$ points), calcium (>7.5 $\mathrm{mg} / \mathrm{dL}, 2$ points $)$ and bicarbonate ( $>19 \mathrm{mEq} / \mathrm{L}, 2$ points). Mortality rate or AKI for patients with a score under 5 was $3 \%$ and with a score over 10 was $59.2 \%$ (25). According to another recent study of 126 patients with rhabdomyolysis, the following variables on admission were independently associated with AKI; peak CK (>12.750 U/L; [odds ratio (OR), 4.9; 95\% CI, 1.416.8]), hypoalbuminemia ( $<33 \mathrm{mg} / \mathrm{dL}$; OR, 5.1; 95\% CI, 1.4-17.7), metabolic acidosis (OR, 5.3; 95\% CI, 1.4-20.3), and decreased PT (<82\%; OR, 4.4; 95\% CI, 1.3-4.5) (16).

The degree of CK elevation correlates with the degree of muscle injury, but it remains unclear if the degree of elevation corresponds to the risk for development of AKI or mortality. In a prospective observational study by Bhavsar and colleagues examined 50 patients with rhabdomyolysis due to trauma and electrical burns. They identified a cutoff value of $\mathrm{CK} 3805 \mathrm{U} / \mathrm{L}$ to be $76.5 \%$ sensitive and $87.9 \%$ specific in predicting AKI after $48 \mathrm{~h}$ of injury (36). In another important study performed by Brown et al reviewed the case records of 1771 trauma patients with increased CK levels. Overall 217 patients (12\%) developed renal failure, with 97 requiring RRT. In this study, peak CK >5000 IU/L was associated with an increased risk of developing AKI (37). The risk of AKI is low when initial CK levels are lower than 15000-20000 IU/L. Lower CK levels may lead to renal injury in patients with sepsis, dehydration, or acidosis (13). On the other hand, in our study, neither initial nor peak value of CK were not correlated with AKI and mortality.

Our findings may help to progress of literature about rhabdomyolysis. However, the study has some limitations. First, this crosssectional study has a relatively small sample size. Therefore, future prospective and multicenter studies with larger sample sizes will be needed to validate our results. Second, the study population consisted of patients at only one center, and therefore, the outcomes may not be applicable to different socioeconomic communities. Third, we could not measure the levels of $\mathrm{Mb}$ in urine and blood. As a result, we could not ascertain the potential interaction effect of $\mathrm{Mb}$ and AKI on the selected outcomes. Fourth, it is not easy to determine accurate and definitive causes of-death in patient with rhabdomyolysis. Another limitation of our study was that we did not discuss the treatment details such as early fluid therapy, mannitol or bicarbonate therapy. Finally and the most important limitation of the current study was that the relatively small sample size may fail to address definitely predictive factors of AKI or need for RRT.

In conclusion, these data clearly demonstrate that the substantial proportion of ED patients with rhabdomyolysis developed the complications of AKI and required RRT. The all-cause mortality rate was relatively high. Increased peak creatinine level, 
increased phosphate level, and prolonged aPTT were found associated with mortality. Early recognition and aggressive fluid replacement should be considered for patients with rhabdomyolysis.

Acknowledgments: This study was presented in the form of oral poster in 4th Eurasian Congress on Emergency Medicine (EACEM), 2014, Antalya, Turkey

Conflict of Interest: The authors declare no potential conflicts of interest with respect to the research, authorship, and/or publication of this article.

\section{References}

1. Warren JD, Blumbergs PC, Thompson PD Rhabdomyolysis: a review. Muscle Nerve 2002;25:332-47.

2. Huerta-Alardín AL, Varon J, Marik PE. Bench-to-bedside review: Rhabdomyolysis - an overview for clinicians. Crit Care 2005;9:158-69.

3. Khan FY. Rhabdomyolysis: a review of the literature. Neth J Med 2009;67:272-83.

4. Bagley WH, Yang H, Shah KH. Rhabdomyolysis. Intern Emerg Med 2007;2:210-8.

5. Giannoglou GD, Chatzizisis YS, Misirli G. The syndrome of rhabdomyolysis: Pathophysiology and diagnosis. Eur $\mathrm{J}$ Intern Med 2007;18:90-100.

6. Elsayed EF, Reilly RF. Rhabdomyolysis: a review, with emphasis on the pediatric population. Pediatr Nephrol 2010;25:7-18.

7. Gabow PA, Kaehny WD, Kelleher SP. The spectrum of rhabdomyolysis. Medicine (Baltimore) 1982;61:141-52.

8. Chatzizisis YS, Misirli G, Hatzitolios AI, Giannoglou GD. The syndrome of rhabdomyolysis: complications and treatment. Eur J Intern Med 2008;19:568-74.

9. Chen CY, Lin YR, Zhao LL, Yang WC, Chang YJ, Wu HP.. Clinical factors in predicting acute renal failure caused by rhabdomyolysis in the ED. Am J Emerg Med 2013;31:1062-6.

10. Oshima Y. Characteristics of drug-associated rhabdomyolysis: analysis of 8,610 cases reported to the U.S. Food and Drug Administration. Intern Med 2011;50:845-53.

11. Campana C, Griffin PL, Simon EL. Caffeine overdose resulting in severe rhabdomyolysis and acute renal failure. Am J Emerg Med 2014;32:111.e3-4.

12. Boutaud O, Roberts LJ II. Mechanism-based therapeutic approaches to rhabdomyolysis-induced renal failure. Free Radic Biol Med 2011;51:1062-7.
13. Bosch X, Poch E, Grau JM. Rhabdomyolysis and acute kidney injury. N Engl J Med 2009; 361:62-72.

14. Holt S, Moore $\mathrm{K}$. Pathogenesis of renal failure in rhabdomyolysis: the role of myoglobin. Exp Nephrol 2000;8:72-6.

15. Heyman SN, Rosen S, Fuchs S, Epstein FH, Brezis M. Myoglobinuric acute renal failure in the rat: a role for medullary hypoperfusion, hypoxia, and tubular obstruction. J Am Soc Nephrol 1996;7:1066-74.

16. Rodríguez E, Soler MJ, Rap O, Barrios C, Orfila MA, Pascual J. Risk factors for acute kidney injury in severe rhabdomyolysis. PLoS One 2013;18:8:e82992.

17. Eyuboglu T, Derinoz O. Rhabdomyolysis due to isoniazid poisoning resulting from the use of intramuscular pyridoxine. Turk J Pediatr 2013;55:328-30.

18. Yilmaz S, Demircioğlu F, Oren H, Güneş B, Irken G. Rhabdomyolysis due to Escherichia coli sepsis in three pediatric patients with acute lymphoblastic leukemia. Pediatr Hematol Oncol 2009;26:57-62.

19. Kasaoka S, Todani M, Kaneko T, Kawamura Y, Oda Y, Tsuruta R, et al. Peak value of blood myoglobin predicts acute renal failure induced by rhabdomyolysis. J Crit Care 2010;25:601-4.

20. Adams BD, Arbogast CB: Rhabdomyolysis. In Adams JG, Barton ED, Collings JL (eds): Emergency Medicine: Clinical Essentials. 2nd ed. Saunders, 2013, pp 1429-38.

21. Mehta RL, Kellum JA, Shah SV, Molitoris BA, Ronco C, Warnock DG, et al. Acute Kidney Injury Network: report of an initiative to improve outcomes in acute kidney injury. Crit Care 2007;11:R31.

22. Macdonald R, Rosner Z, Venters H. Case series of exercise-induced rhabdomyolysis in the New York City jail system. Am J Emerg Med. 2014;32:466-7.

23. Zimmerman JL, Shen MC. Rhabdomyolysis. Chest 2013;144:1058-65.

24. Graves EJ, Gillum BS. Detailed diagnoses and procedures, National Hospital Discharge Survey, 1995. Vital Health Stat 1997 Nov;(130):1-146.

25. McMahon GM, Zeng X, Waikar SS. A risk prediction score for kidney failure or mortality in rhabdomyolysis. JAMA Intern Med 2013;173:1821-8.

26. Mannix R, Tan ML, Wright R, Baskin M.. Acute pediatric rhabdomyolysis: causes and rates of renal failure. Pediatrics 2006;118:2119-25.

27. Luck RP, Verbin S. Rhabdomyolysis: a review of clinical presentation, etiology, diagnosis, and management. Pediatr Emerg Care 2008;24:262-8. 
28. Holmes JF, McGahan JP, Wisner DH. Rate of intraabdominal injury after a normal abdominal computed tomographic scan in adults with blunt trauma. Am J Emerg Med 2012;30:574-9.

29. Yanturali S, Cete Y, Oktay C, Eray O, Atilla R, Cevik AA, et al. Diagnostic value of intraabdominal pressure measurement in blunt abdominal trauma patients. Turk $\mathrm{J}$ Emerg Med 2004;4:105-8

30. Baydin A, Yardan T, Guven H, Dervisoglu A, Otal Y, Eden AO, et al. The relation of the lactate, base excess and Injury Severity Scores with mortality in trauma. Turk J Emerg Med 2007;7:97-101.

31. Dur A, Cander B, Koçak S, Girisgin S, Gul M, Koyuncu F. Multiple trauma patients and trauma scoring systems in emergency-intensive care unit. JAEM 2009;8:24-7.

32. Altunci YA, Aldemir M, Guloglu C, Ustundag M, Orak M.. The effective factors in emergency department observation on hospitalization requirement and mortality in blunt trauma patients. JAEM 2010:9:117-20.

33. Fernandez WG, Hung O, Bruno GR, Galea S, Chiang WK Factors predictive of acute renal failure and need for hemodialysis among ED patients with rhabdomyolysis. Am J Emerg Med 2005;23:-7.
34. Delaney KA, Givens ML, Vohra RB. Use of RIFLE criteria to predict the severity and prognosis of acute kidney injury in emergency department patients with rhabdomyolysis. J Emerg Med 2012;42:521-8.

35. Talving P, Karamanos E, Skiada D, Lam L, Teixeira PG, Inaba $\mathrm{K}$, et al. Relationship of creatine kinase elevation and acute kidney injury in pediatric trauma patients. J Trauma Acute Care Surg 2013;74:912-6.

36. Bhavsar P, Rathod KJ, Rathod D, Chamania CS. Utility of Serum Creatinine, Creatine Kinase and Urinary Myoglobin in Detecting Acute Renal Failure due to Rhabdomyolysis in Trauma and Electrical Burns Patients. Indian J Surg 2013;75:17-21

37. Brown CV, Rhee P, Chan L, Evans K, Demetriades D, Velmahos GC. Preventing renal failure in patients with rhabdomyolysis: do bicarbonate and mannitol make a difference? J Trauma 2004;56:1191-96

Copyright (C) 2014 The Author(s); This is an open-access article distributed under the terms of the Creative Commons Attribution License (http://creativecommons.org/licenses/by/4.0), which permits unrestricted use, distribution, and reproduction in any medium, provided the original work is properly cited. All Rights reserved by international journal of Medical Science and Discovery. 